\title{
Two-Dimensional Temperature Field Distribution Reconstruction Based on Least Square Method and Radial Basis Function Approximation
}

\author{
Ruixi Jia $^{1,2}$ and Qingyu Xiong ${ }^{1,3}$ \\ ${ }^{1}$ Key Laboratory of Dependable Service Computing in Cyber Physical Society, MOE, Chongqing University, \\ Chongqing 400044, China \\ ${ }^{2}$ School of Automation, Chongqing University, Chongqing 400044, China \\ ${ }^{3}$ School of Software Engineering, Chongqing University, Chongqing 400044, China
}

Correspondence should be addressed to Qingyu Xiong; xiong03@cqu.edu.cn

Received 2 May 2017; Revised 29 May 2017; Accepted 6 September 2017; Published 19 October 2017

Academic Editor: Sebastian Heidenreich

Copyright (C) 2017 Ruixi Jia and Qingyu Xiong. This is an open access article distributed under the Creative Commons Attribution License, which permits unrestricted use, distribution, and reproduction in any medium, provided the original work is properly cited.

\begin{abstract}
The information of temperature field distribution is complex but quite important for theoretical study and industrial applications, such as burning, drying, and heating, because it can reflect the internal running state of industrial equipment, assist to develop control strategy, and ensure safety in operation. Acoustic imaging is a kind of noncontact temperature measurement technique which is able to achieve temperature field distribution reconstruction. The process of temperature field distribution reconstruction using acoustic imaging is an inverse problem and its reconstruction performance depends on reconstruction algorithm. In this paper, a modified reconstruction algorithm is investigated. Compared with other reconstruction methods of least square algorithm and radial basis function approximation, the reconstruction results indicate that the modified reconstruction algorithm occupies the best information reflection of temperature field distribution and the reconstruction accuracy is improved.
\end{abstract}

\section{Introduction}

The information of temperature field distribution is complex but of crucial important for some industrial applications, such as burning, drying, and heating, because it can reflect the internal running state of industrial equipment, assist to develop control strategy, and ensure safety in operation [1-3]. Thus, it becomes a critical issue to explore a proper temperature measurement method to reconstruct temperature field distribution and acquire temperature information in industrial environment.

Common temperature measurement techniques include contact and noncontact measurement methods. Contact measurement techniques, for instance, thermal thermocouples, thermal resistances, mercury thermometers, and fiber pyrometer, are hardly applied to accomplish temperature field distribution reconstruction, since these methods are only suitable for single-point temperature measurement [4-7].
Noncontact measurements mainly consist of laser spectroscopy, infrared temperature measurement, and optical temperature measurement. Though, noncontact measurements surmount the limitation of contact measurements in single-point temperature measurement, these techniques are only applied to the temperature measurement of material surface [8]. In addition, the measurement accuracy of infrared radiations may be deteriorated because of different emissivity and reflectivity of infrared radiations from other sources [9, 10]. The measurement result of optical temperature measurement could be affected because the camera lens is disturbed by dust.

Acoustic imaging is a kind of noncontact temperature measurement technique which is able to achieve temperature field distribution reconstruction. The theory of this technique is in accordance with the relevance between acoustic velocity and temperature [11]. Reconstructing temperature field distribution using acoustic imaging is an inverse process which 
depends on reconstruction algorithm. One of the most widely used reconstruction algorithms is least square algorithm (LSA) [12-14]. But, this algorithm could not complete an entire temperature field distribution reconstruction due to its flaw of interpolation range. So, temperature message on measuring edge may be ignored. In order to overcome the shortage of LSA, [4] proposes a reconstruction algorithm based on radial basis function approximation (RBF) and singular values decomposition. However, LSA has advantages of relatively high stability and reconstruction accuracy and $\mathrm{RBF}$ is capable of mathematic fitting. Reconstruction results would be a breakthrough if the advantages of LSA and RBF are considered and combined reasonably. In this paper, a modified reconstruction algorithm based on LAS and RBF is investigated. Compared with LSA and RBF, the reconstruction results indicate that the modified reconstruction algorithm occupies the best information reflection of temperature field distribution and the reconstruction accuracy is improved.

This paper is organized as follows. Section 2 describes the basic principle of acoustic imaging. Section 3 presents the modified temperature field distribution reconstruction algorithm. Section 4 uses three temperature field distribution models to validate the performance of proposed reconstruction algorithm and shows simulation experiment results and analysis. Section 5 focuses on conclusions and future research.

\section{The Basic Principle of Acoustic Imaging}

The fundamental theory of acoustic imaging is that the acoustic speed in a medium is a function of the medium temperature. The simplest acoustic imaging is single path temperature measurement. It consists of two acoustic transducers installed on both sides of a path where acoustic travels. The acoustic transducers are used to transmit acoustic signal and detect it, respectively. The relationship between acoustic velocity and temperature is expressed as [14]

$$
c=\sqrt{k \frac{R}{M_{g}} T}=Z \sqrt{T}
$$

where $c$ is acoustic velocity, $R$ is universal gas constant, $T$ is absolute temperature (K), and $k$ and $M_{g}$ are ratio and average molecular weight of gas. For different gas, $k, R$, and $M_{g}$ are fixed constants. So, they are replaced by a coefficient $Z$. If medium is air, $k, R$, and $M_{g}$ are $1.40,8314$, and 29. Thus, $Z$ equals 20.03 .

On the contrary, if acoustic speed $c$ and the type of gas are known, then the temperature $T$ is expressed as

$$
T=\left(\frac{c}{Z}\right)^{2}
$$

The distance $L$ between acoustic transducers could be measured and acquired. According to the correlativity of acoustic speed $c$, acoustic flying time (AFT) $t_{k}$, and distance $L$, we can get

$$
c=\frac{L}{t_{k}} .
$$

Combining (2) and (3), $T$ could be rewritten as

$$
T=\frac{1}{Z^{2}}\left(\frac{L}{t_{k}}\right)^{2}-273.15
$$

From (4), the average temperature $\left({ }^{\circ} \mathrm{C}\right)$ of the measuring path is gained.

\section{The Modified Temperature Field Distribution Reconstruction Algorithm}

3.1. Least Square Algorithm. The single path temperature measurement of acoustic imaging barely fulfills temperature field distribution. But, if several acoustic transducers are installed appropriately in a measuring area, multiple paths acoustic travels will form and more temperature information will be gained. Then, proper reconstruction algorithm can reconstruct the temperature field distribution of measuring area.

Figure 1 shows a specific acoustic transducer layout in a square measuring area $(10 \mathrm{~m} \times 10 \mathrm{~m})$ isometrically divided into 100 blocks by dashed lines. The geometric center of square measuring area is defined as the origin of coordinate. The acoustic transducers $S_{0}-S_{11}$ are represented by black symbols. The working procedure of acoustic transducers is as follows: first, transducer $S_{0}$ acts as a transmitter and launches acoustic signal and transducers $S_{4}, S_{5}, \ldots, S_{8}$ receive the signal. Then, transducer $S_{1}$ launches acoustic signal and transducers $S_{4}, S_{5}, \ldots, S_{11}$ detect the signal. This process will not end until all the transducers launch acoustic signal and receive the signal. There are 42 acoustic flying routes (AFR). Temperature field distribution in the measuring field could be reconstructed with estimated AFTs in 42 AFRs and reconstruction algorithm.

Theoretically, the $k$ th AFT $t_{k}^{\prime}$ in the $k$ th AFR $l_{k}$ is described as [13]

$$
t_{k}^{\prime}=\int_{l_{k}} a d s=\int_{l_{k}} \frac{1}{B \sqrt{T(x, y)}} d s,
$$

where $a_{i}$ is the reciprocal of acoustic velocity in the $i$ th specific block. $T(x, y)$ is temperature field distribution model which is artificially designed. The function of reconstruction algorithm is to reconstruct the model $T(x, y)$.

Assuming that the measuring area in Figure 1 is divided into $N$ blocks, and temperature distribution in each block is uniform, (5) is turned into

$$
t_{k}^{\prime}=\sum_{i}^{N} \Delta S_{k i} a_{i}
$$

where $k$ and $i$ represent the number of paths and the number of blocks. $\Delta S_{k i}$ indicates the length of the $k$ th AFR passing through the ith block. 


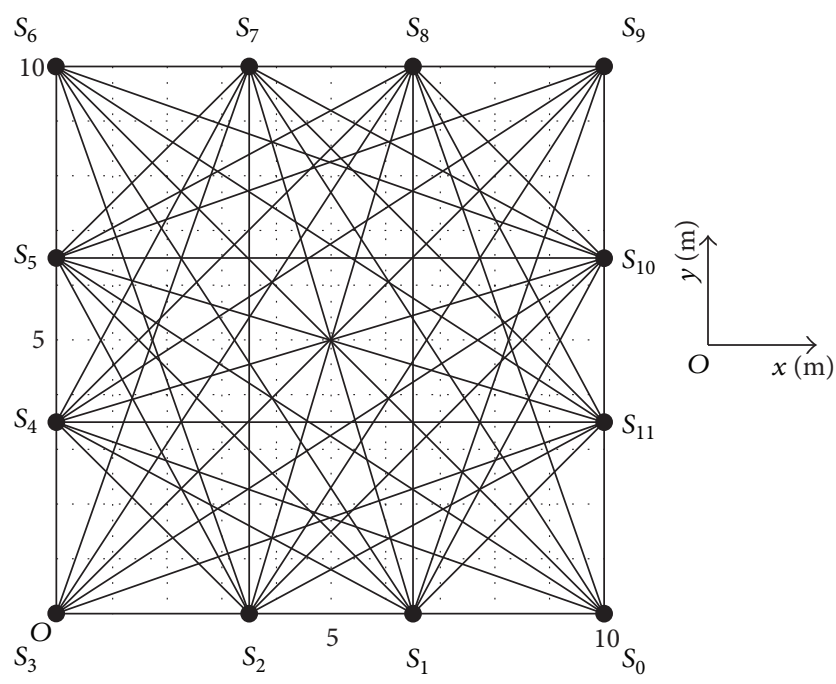

FIGURE 1: A specific acoustic transducer layout in a square measuring area.

If $t_{k}$ is the practical measured value of AFT in the $k$ th AFR, the difference $\varepsilon_{k}$ between $t_{k}$ and the theoretical AFT $t_{k}^{\prime}$ is expressed as

$$
\varepsilon_{k}=t_{k}-t_{k}^{\prime}=t_{k}-\sum_{i}^{N} \Delta S_{k i} a_{i}
$$

Supposing $M$ is the amount of AFRs, thus, there are $M$ differences $\varepsilon_{k}$ in total. In order to make $t_{k}^{\prime}$ and $t_{k}$ the same as far as possible, according to LSA, $\varepsilon_{k}$ should be minimized, which means minimizing the sum of the squares of these $M$ differences $\varepsilon_{k}$, given by

$$
\frac{\partial}{\partial a_{i}} \sum_{k=1}^{M}\left(t_{k}-\sum_{i}^{N} \Delta S_{k i} a_{i}\right)^{2}=0 .
$$

Then, canonical equation is gained as

$$
S^{T} S A=S^{T} t
$$

where

$$
\begin{aligned}
A & =\left[\begin{array}{llll}
a_{1} & a_{2} & \cdots & a_{N}
\end{array}\right]^{T} \\
t & =\left[\begin{array}{llll}
t_{1} & t_{2} & \cdots & t_{M}
\end{array}\right]^{T} \\
S & =\left[\begin{array}{cccc}
\Delta S_{11} & \Delta S_{12} & \cdots & \Delta S_{1 N} \\
\Delta S_{21} & \Delta S_{22} & \cdots & \Delta S_{2 N} \\
\cdots & \cdots & \cdots & \cdots \\
\Delta S_{M 1} & \Delta S_{M 2} & \cdots & \Delta S_{M N}
\end{array}\right] .
\end{aligned}
$$

So, the reciprocal matrix $A$ of average acoustic velocity in each block can be calculated as

$$
A=\left(S^{T} S\right)^{-1} S^{T} t
$$

As described above, when the geometric features of measuring area and the arrangement of acoustic transducers are set, $S$ becomes a constant matrix which can be determined beforehand. Moreover, when the temperature field distribution model is designed, $t$ can be obtained from (5). Thus, the average temperature array of the blocks $T$ is estimated as

$$
T=\frac{1}{A^{2} Z^{2}},
$$

where $T=\left[\begin{array}{llll}T_{1} & T_{1} & \cdots & T_{N}\end{array}\right]^{T}$ and $N$ is 100 .

When the average temperature of each block is worked out, temperature field distribution of the measuring area could be reconstructed by interpolation among $N$ values in matrix $T$. However, temperature information over the border of the measuring field (Figure 1) could be missing because of the interpolation limitation of LSA.

3.2. Modified Temperature Field Distribution Reconstruction Algorithm Based on LSA and RBF. To make up for deficiencies of LSA in interpolation and meanwhile make use of its advantages in stability and reconstruction accuracy, a modified temperature field distribution reconstruction algorithm based on LSA and RBF is proposed.

In Figure 1, assuming that $\left(x_{m}, y_{m}\right)$ is the coordinate of the central point in the $m$ th block, the RBF of this block is expressed as

$$
\varphi_{m}(x, y)=e^{-\theta \sqrt{\left(x-x_{m}\right)^{2}+\left(y-y_{m}\right)^{2}}}
$$

where $\theta$ is the shape parameter of RBF and $\theta$ equals 0.0001 .

Temperature field distribution model $T(x, y)$ could be indicated with a linear combination of $N$ RBFs and shown as

$$
T(x, y)=\sum_{m=1}^{N} \omega_{m} \varphi_{m}(x, y)
$$

If each element of matrix $T$ in (14) is the central point temperature of corresponding block, then, matrix $T$ could be displayed as

$$
\begin{gathered}
T_{1}=\sum_{m=1}^{N} \varepsilon_{m} \varphi_{m}\left(x_{1}, y_{1}\right) \\
T_{2}=\sum_{m=1}^{N} \varepsilon_{m} \varphi_{m}\left(x_{2}, y_{2}\right) \\
\vdots \\
T_{N}=\sum_{m=1}^{N} \varepsilon_{m} \varphi_{m}\left(x_{N}, y_{N}\right) .
\end{gathered}
$$

Equation (15) is rewritten as

$$
T=\Phi \omega,
$$




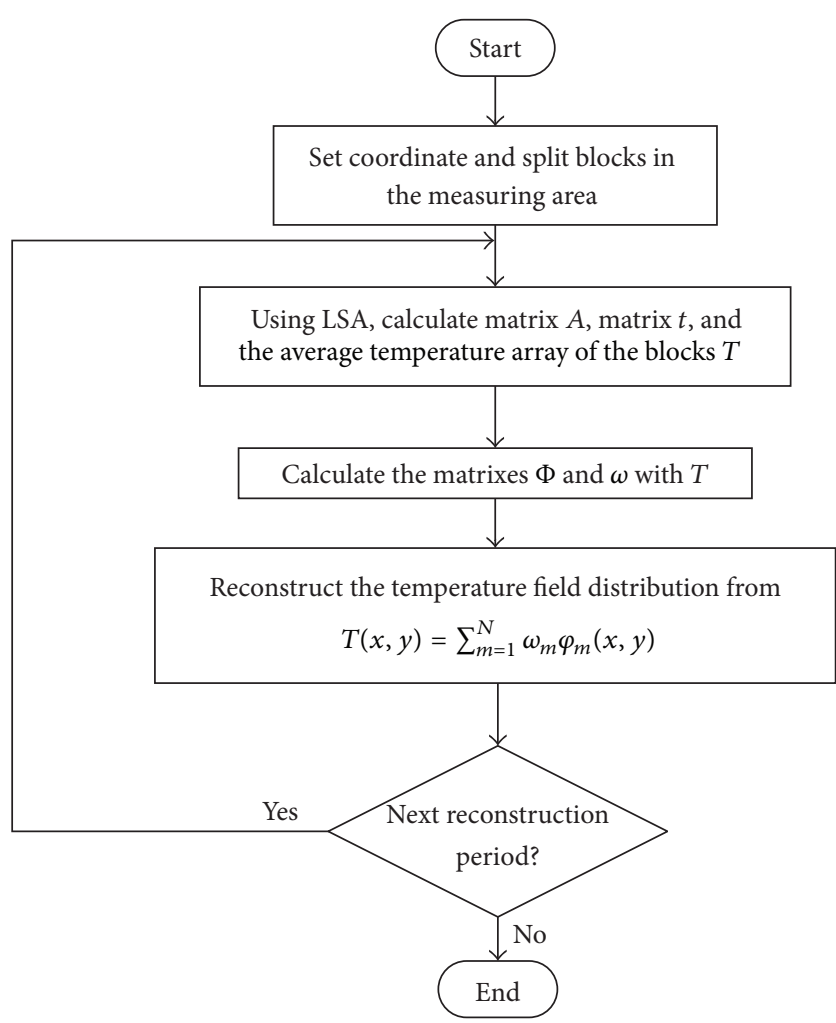

FIGURE 2: The procedure of modified reconstruction algorithm based on LSA and RBF.

where

$$
\begin{aligned}
& \omega=\left[\begin{array}{llll}
\omega_{1} & \omega_{1} & \cdots & \omega_{N}
\end{array}\right]^{T}, \\
& =\left\{\begin{array}{lccc}
\varphi_{1}\left(x_{1}, y_{1}\right) & \varphi_{2}\left(x_{1}, y_{1}\right) & \ldots & \varphi_{N}\left(x_{1}, y_{1}\right) \\
\varphi_{1}\left(x_{2}, y_{2}\right) & \varphi_{2}\left(x_{2}, y_{2}\right) & \ldots & \varphi_{N}\left(x_{2}, y_{2}\right) \\
\cdots & \ldots & \cdots & \ldots \\
\varphi_{1}\left(x_{N}, y_{N}\right) & \varphi_{2}\left(x_{N}, y_{N}\right) & \cdots & \varphi_{N}\left(x_{N}, y_{N}\right) .
\end{array}\right.
\end{aligned}
$$

When the coordinate of measuring area is set, matrix $\Phi$ would be calculated ahead of time and the matrix $T$ could be gained by LSA. Thus, the unknown matrix $\omega$ is acquired as

$$
\omega=\Phi^{-1} T \text {. }
$$

In the end, the reconstructed temperature field distribution could be accomplished from (14) with $N$ estimated $\omega$. The procedure of modified reconstruction algorithm based on LSA and RBF is demonstrated in Figure 2.

\section{Simulation Experiment Results and Analysis}

A reconstruction algorithm should reconstruct multifarious temperature field distributions with complexity levels. Using MATLAB software and air as simulation experiment platform and medium, three temperature field distribution models with different hot zones distributed over the measuring area in Figure 1 are artificially made and expressed as one-peak symmetrical temperature field distribution model with one hot zone:

$$
T M_{1}(x, y)=800+1100 \sin \left(\frac{\pi}{10} x\right) \sin \left(\frac{\pi}{10} y\right),
$$

two-peak asymmetrical temperature field distribution model with two hot zones:

$$
\begin{aligned}
\operatorname{TM}_{2}(x, y)= & 800+1100 e^{\left[-21(x / 12-3 / 7)^{2}-21(y / 11-1 / 5)^{2}\right]} \\
& +1100 e^{\left[-21(x / 10-3 / 7)^{2}-21(y / 9-9 / 10)^{2}\right]}
\end{aligned}
$$

and three-peak asymmetrical temperature field distribution model with three hot zones:

$$
\begin{aligned}
\operatorname{TM}_{3}(x, y)= & 800+1100 e^{\left[-20(x / 9-1 / 5)^{2}-20(y / 9-5 / 7)^{2}\right]} \\
& +1000 e^{\left[-20(x / 9-2 / 5)^{2}-14(y / 9-1 / 4)^{2}\right]} \\
& +1100 e^{\left[-14(x / 10-4 / 5)^{2}-13(y / 11-4 / 7)^{2}\right]}
\end{aligned}
$$

The simulation experiment results are displayed using LSA, $\mathrm{RBF}$, and the modified algorithm with two-dimensional isothermal contour figures in Figures 3-5.

From Figures 3-5, RBF and the modified algorithm overcome the disadvantage of LSA in interpolation limitation and offer entire temperature field information of the measuring area. But, the modified algorithm could present a better and more precise reconstruction performance over the border of the measuring area than LSA.

When the reconstruction process is finished, quantitative analysis of reconstruction performance which consists of mean relative error $E_{m}$ and root-mean-square percent error $E_{r}$ is computed. $E_{m}$ and $E_{r}$ are defined as

$$
\begin{aligned}
E_{m} & =\frac{1}{n} \sum_{j=1}^{n}\left|\frac{T_{R j}-T_{M j}}{T_{M j}}\right| \times 100 \% \\
E_{r} & =\frac{\sqrt{(1 / n) \sum_{j=1}^{n}\left(T_{R j}-T_{M j}\right)^{2}}}{T_{M_{\text {mean }}}} \times 100 \%,
\end{aligned}
$$

where $n$ is the number of calculating points in the measuring area. At the coordinate $\left(x_{j}, y_{j}\right), T_{R j}$ is from reconstructed temperature field distribution with LSA, RBF, and the modified algorithm and $T_{M j}$ is from model. $T_{M \text { mean }}$ is the mean temperature value of the temperature field distribution model.

It is important to monitor the hotspots in hot zones because they may lead to unexpected and major accidents. Hotspot temperature error $E_{h}$ is given as

$$
E_{h}=\left|\frac{T_{R h}-T_{M h}}{T_{M h}}\right| \times 100 \%,
$$




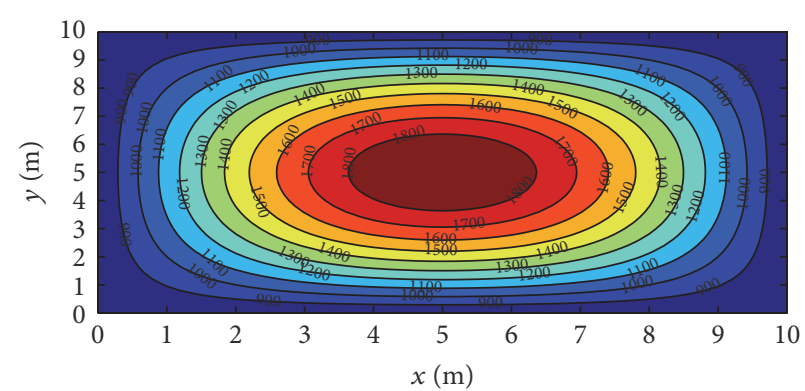

(a)

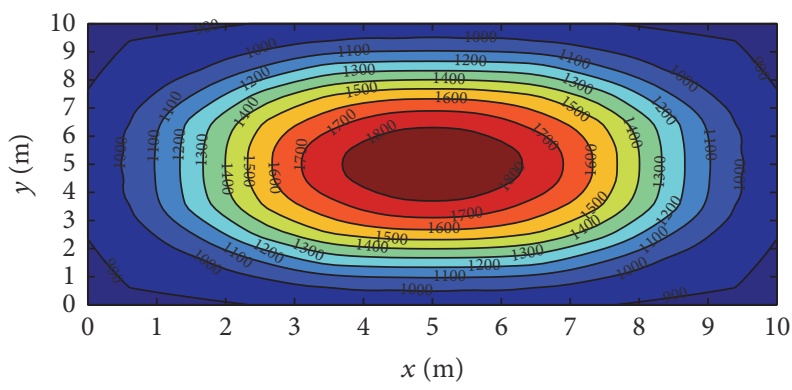

(c)

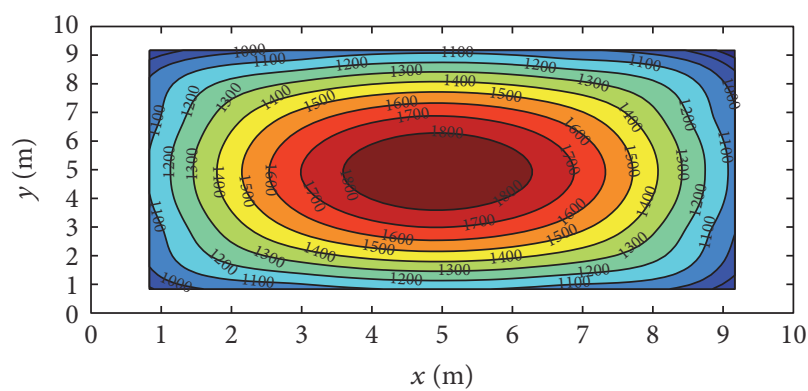

(b)

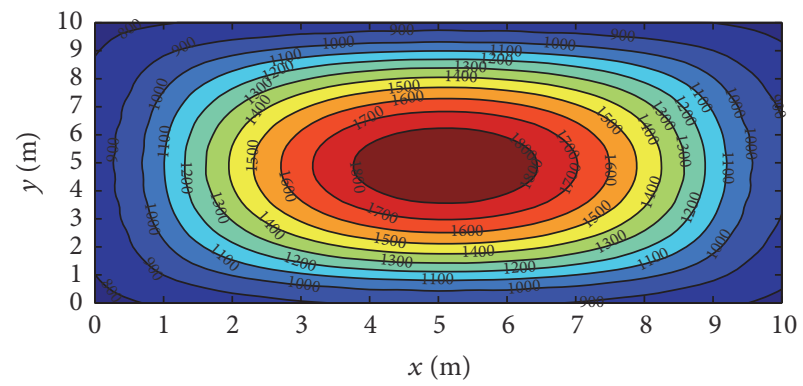

(d)

FIGURE 3: The comparison of simulation experiment results of one-peak symmetrical temperature field distribution: (a) model; (b) with LSA; (c) with RBF; (d) with modified algorithm.

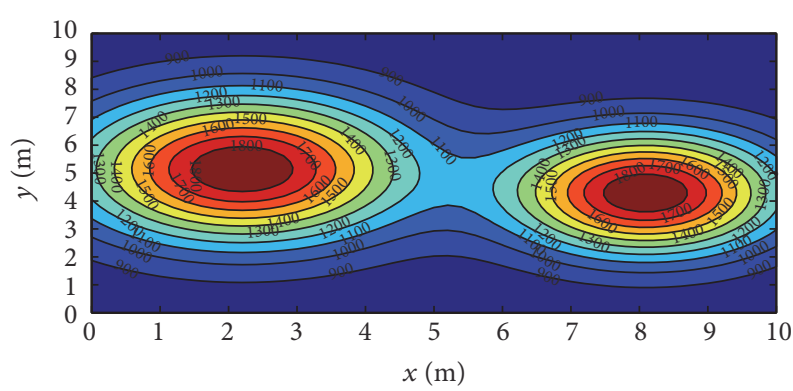

(a)

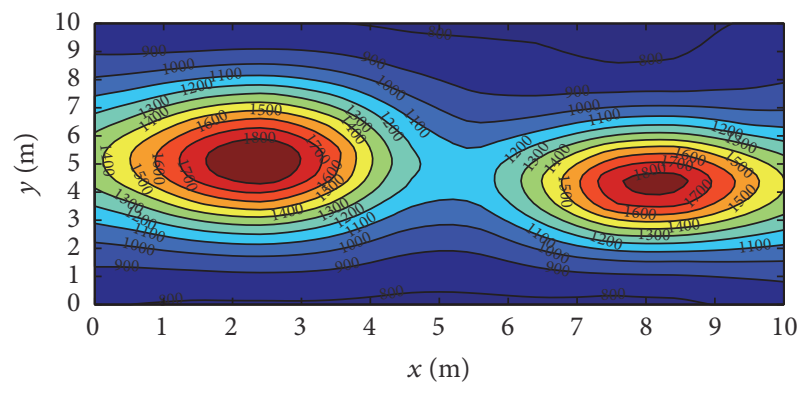

(c)

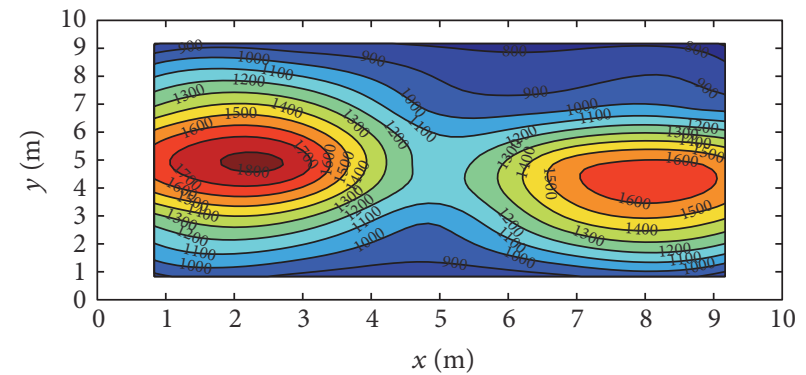

(b)

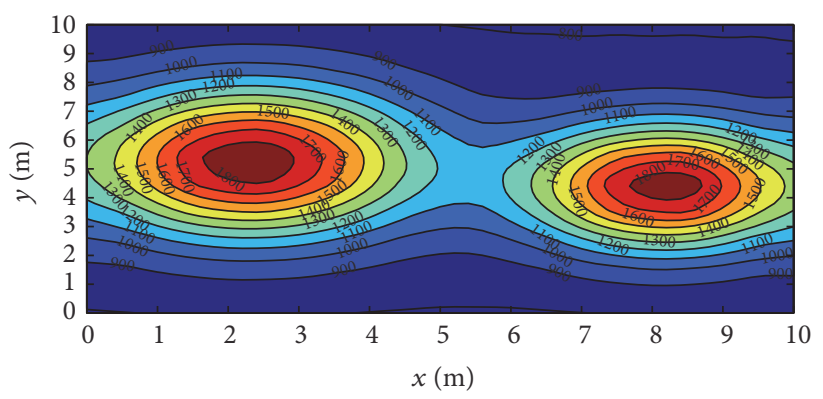

(d)

FIGURE 4: The comparison of simulation experiment results of two-peak asymmetrical temperature field distribution: (a) model; (b) with LSA; (c) with RBF; (d) with modified algorithm.

where $T_{M h}$ and $T_{R h}$ are the temperature values of hotspots from the temperature field distribution models and the reconstructed ones.

Tables 1-3 show error analysis of $E_{m}, E_{r}$, and $E_{h}$.
Judging from Tables 1 and 2, for simple temperature field distribution, like one-symmetrical, though $E_{m}$ and $E_{r}$ with LSA and RBF are small $\left(E_{m}: 4.28 \%\right.$ and $4.50 \%$, $E_{r}: 5.01 \%$, and $4.92 \%$ ), the modified algorithm owns the 


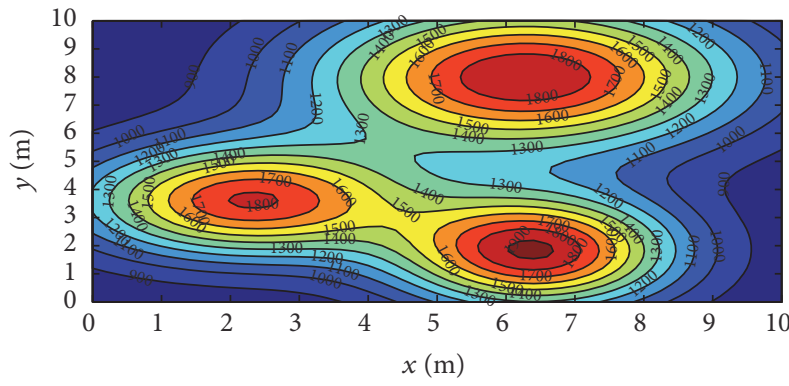

(a)

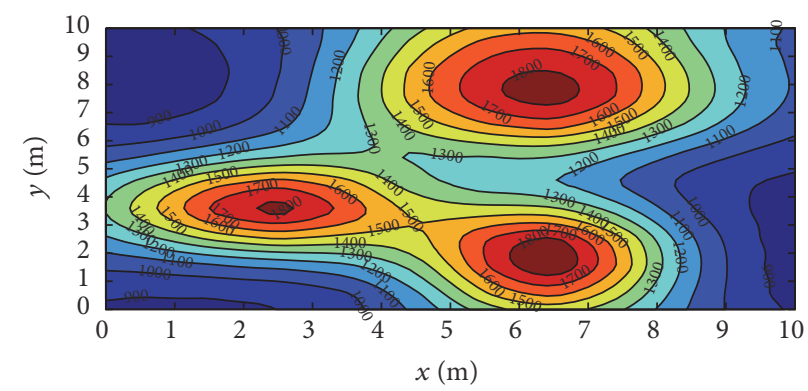

(c)

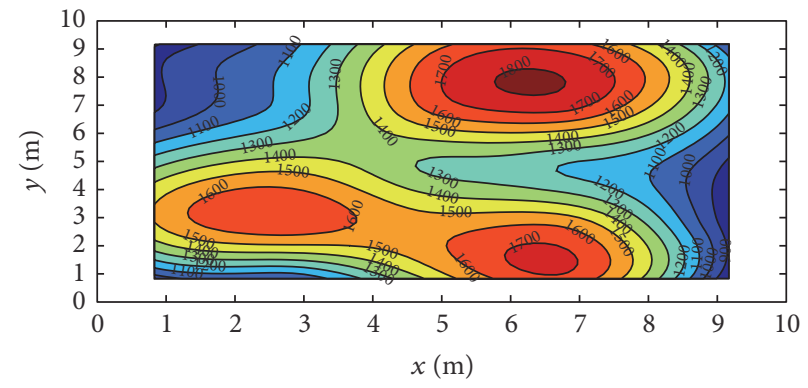

(b)

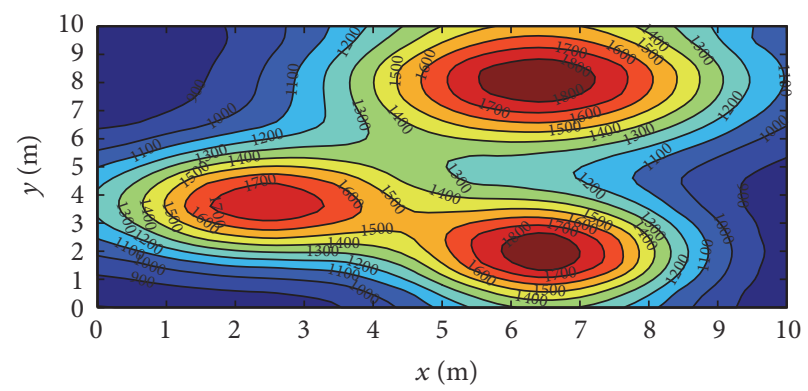

(d)

FIGURE 5: The comparison of simulation experiment results of three-peak asymmetrical temperature field distribution: (a) model; (b) with LSA; (c) with RBF; (d) with modified algorithm.

TABLE 1: Error analysis of mean relative error $E_{m}(\%)$.

\begin{tabular}{lllc}
\hline & LSA & RBF & The modified algorithm \\
\hline One-peak symmetrical temperature distribution & 4.28 & 4.50 & 3.89 \\
Two-peak asymmetrical temperature distribution & 6.47 & 5.22 & 4.54 \\
Three-peak asymmetrical temperature distribution & 8.46 & 6.62 & 5.67 \\
\hline
\end{tabular}

TABLE 2: Error analysis of mean relative error $E_{r}(\%)$.

\begin{tabular}{lccc}
\hline & LSA & RBF & The modified algorithm \\
\hline One-peak symmetrical temperature distribution & 5.01 & 4.92 & 4.26 \\
Two-peak asymmetrical temperature distribution & 7.33 & 6.03 & 5.51 \\
Three-peak asymmetrical temperature distribution & 10.09 & 7.48 & 6.48 \\
\hline
\end{tabular}

TABLE 3: Error analysis of hotspot temperature error $E_{h}$.

\begin{tabular}{|c|c|c|c|c|c|c|c|}
\hline \multirow{2}{*}{$\begin{array}{l}\text { Reconstructed } \\
\text { temperature } \\
\text { distributions }\end{array}$} & \multicolumn{4}{|c|}{ Temperature values of hotspots $\left({ }^{\circ} \mathrm{C}\right)$} & \multicolumn{3}{|c|}{ The hotspot temperature error $E_{h}(\%)$} \\
\hline & Models & LSA & $\mathrm{RBF}$ & The modified algorithm & LSA & $\mathrm{RBF}$ & The modified algorithm \\
\hline $\begin{array}{l}\text { One-peak } \\
\text { symmetrical }\end{array}$ & 1976.47 & 1929.23 & 1923.11 & 1940.56 & 2.39 & 2.70 & 1.82 \\
\hline \multirow{2}{*}{$\begin{array}{l}\text { Two-peak } \\
\text { asymmetrical }\end{array}$} & 1953.68 & 1853.26 & 1874.56 & 1891.63 & 5.14 & 4.05 & 3.18 \\
\hline & 1940.22 & 1821.48 & 1865.13 & 1877.39 & 6.12 & 3.87 & 3.24 \\
\hline \multirow{3}{*}{$\begin{array}{l}\text { Three-peak } \\
\text { asymmetrical }\end{array}$} & 1870.01 & 1726.77 & 1785.49 & 1805.50 & 7.66 & 4.52 & 3.45 \\
\hline & 1835.36 & 1671.28 & 1760.29 & 1773.37 & 8.94 & 4.09 & 3.38 \\
\hline & 1961.58 & 1758.95 & 1893.12 & 1904.81 & 10.33 & 3.49 & 2.90 \\
\hline
\end{tabular}


relatively best properties of $E_{m}$ and $E_{r}\left(E_{m}: 3.89 \%, E_{r}\right.$ : $4.26 \%)$. When the complexity of temperature field distribution increases, such as two-peak and three-peak asymmetrical, the modified algorithm also possesses the relatively best performance of $E_{m}$ and $E_{r}$ among LSA and RBF $\left(E_{m}: 5.67 \%\right.$, $\left.E_{r}: 6.48 \%\right)$. For hotspot temperature error $E_{h}$ in Table 3, the measured temperature values of hotpots with the modified algorithm are the closest to models' compared with LSA and $\mathrm{RBF}$ and the reconstruction accuracy is relatively improved (one-symmetrical: $1940.56^{\circ} \mathrm{C}, E_{h}: 1.82 \%$; two-asymmetrical: $1891.63^{\circ} \mathrm{C}$ and $1877.39^{\circ} \mathrm{C}, E_{h}: 3.18 \%$ and $3.24 \%$; three-asymmetrical: $1785.49^{\circ} \mathrm{C}, 1760.29^{\circ} \mathrm{C}$, and $1893.12^{\circ} \mathrm{C}, E_{h}: 3.48 \%$, $3.38 \%$, and $2.90 \%$ ).

In a word, comparisons of reconstructed two-dimensional isothermal contour figures and error analysis of $E_{m}$, $E_{r}$, and $E_{h}$ indicate that the modified algorithm occupies the best information reflection of temperature field distribution and the highest reconstruction accuracy.

\section{Conclusions and Future Research}

The information of temperature field distribution is complex but quite important for theoretical study and industrial applications. Temperature field distribution reconstruction using acoustic imaging is an inverse problem and its reconstruction performance depends on reconstruction algorithm. In this paper, a modified reconstruction algorithm based on least square method and radial basis function approximation is investigated. Firstly, the basic principle of acoustic is described. Secondly, the modified algorithm is expressed. Finally, simulation experiments are carried out to study the reconstruction performance of the proposed algorithm. Compared with LSA and RBF, the results of simulation experiments show that the modified reconstruction algorithm occupies the best information reflection of temperature field distribution and the highest reconstruction accuracy.

The major work of future research should focus on achievement of actual experimental system. The measuring area could be a square cavity with acoustic transducers installed in a specific plane of it. The hot zones maybe replaced by candles or heater source. Simultaneously, (5) is not fit for calculating AFT because temperature field distribution of the practical experiment environment is hardly artificially made. Therefore, a method for estimating AFT based on acoustic transmitted and received waveforms should be studied. Thus, with actual experimental system and AFT estimation method, the advantages of the proposed reconstruction algorithm over LSA and RBF will be verified.

\section{Conflicts of Interest}

The authors declare that there are no conflicts of interest regarding the publication of this paper.

\section{Acknowledgments}

This work is supported by Major State Basic Research Development Program (973 Program Grant no. 2013CB328903).

\section{References}

[1] M. Bramanti, E. A. Salerno, A. Tonazzini, S. Pasini, and A. Gray, "An acoustic pyrometer system for tomographic thermal imaging in power plant boilers," IEEE Transactions on Instrumentation and Measurement, vol. 45, no. 1, pp. 159-167, 1996.

[2] M. Pöschl and T. Sattelmayer, "Influence of temperature inhomogeneities on knocking combustion," Combustion and Flame, vol. 153, no. 4, pp. 562-573, 2008.

[3] X. Shen, Q. Xiong, X. Shi, K. Wang, S. Liang, and M. Gao, "Ultrasonic temperature distribution reconstruction for circular area based on Markov radial basis approximation and singular value decomposition," Ultrasonics, vol. 62, pp. 174-185, 2015.

[4] R. Jia, Q. Xiong, and S. Liang, "Acoustic imaging for temperature distribution reconstruction," AIP Advances, vol. 6, no. 12, Article ID 125018, 2016.

[5] C. Lou and H.-C. Zhou, "Deduction of the two-dimensional distribution of temperature in a cross section of a boiler furnace from images of flame radiation," Combustion and Flame, vol. 143, no. 1-2, pp. 97-105, 2005.

[6] W.-Y. Tsai, H.-C. Chen, and T.-L. Liao, "High accuracy ultrasonic air temperature measurement using multi-frequency continuous wave," Sensors and Actuators A: Physical, vol. 132, no. 2, pp. 526-532, 2006.

[7] A. Kosugi, I. Ihara, and I. Matsuya, "Accuracy evaluation of surface temperature profiling by a laser ultrasonic method," Japanese Journal of Applied Physics, vol. 51, no. 7S, p. 07GB01, 2012.

[8] R. Jia, Q. Xiong, L. Wang et al., "Study of ultrasonic thermometry based on ultrasonic time-of-flight measurement," AIP Advances, vol. 6, no. 3, Article ID 035006, 2016.

[9] D. Stuerga and P. Gaillard, "Microwave heating as a new way to induce localized enhancements of reaction rate. Non-isothermal and heterogeneous kinetics," Tetrahedron, vol. 52, no. 15, pp. 5505-5510, 1996.

[10] T. Durka, G. D. Stefanidis, T. van Gerven, and A. Stankiewicz, "On the accuracy and reproducibility of fiber optic (FO) and infrared (IR) temperature measurements of solid materials in microwave applications," Measurement Science and Technology, vol. 21, no. 4, Article ID 045108, 2010.

[11] R. Jia, Q. Xiong, K. Wang, L. Wang, G. Xu, and S. Liang, "The study of three-dimensional temperature field distribution reconstruction using ultrasonic thermometry," AIP Advances, vol. 6, no. 7, Article ID 075007, 2016.

[12] G. D'Antona and N. Seifnaraghi, "Temperature distribution reconstruction by eigenfunction interpolation of boundary measurement data," IEEE Transactions on Instrumentation and Measurement, vol. 63, no. 2, article no. 16, pp. 334-342, 2014.

[13] X. Shen, Q. Xiong, W. Shi, S. Liang, X. Shi, and K. Wang, "A new algorithm for reconstructing two-dimensional temperature distribution by ultrasonic thermometry," Mathematical Problems in Engineering, vol. 2015, Article ID 916741, 10 pages, 2015.

[14] R. Jia, Q. Xiong, G. Xu, K. Wang, and S. Liang, "A method for two-dimensional temperature field distribution reconstruction," Applied Thermal Engineering, vol. 111, pp. 961-967, 2017. 


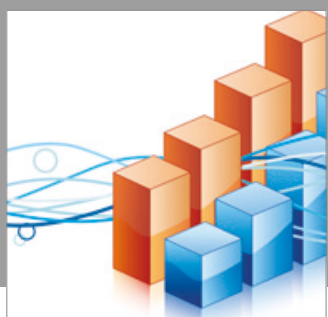

Advances in

Operations Research

vatersals

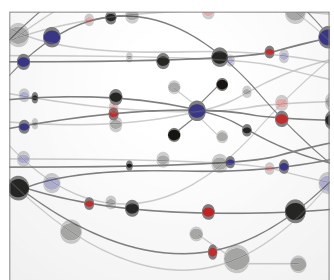

\section{The Scientific} World Journal
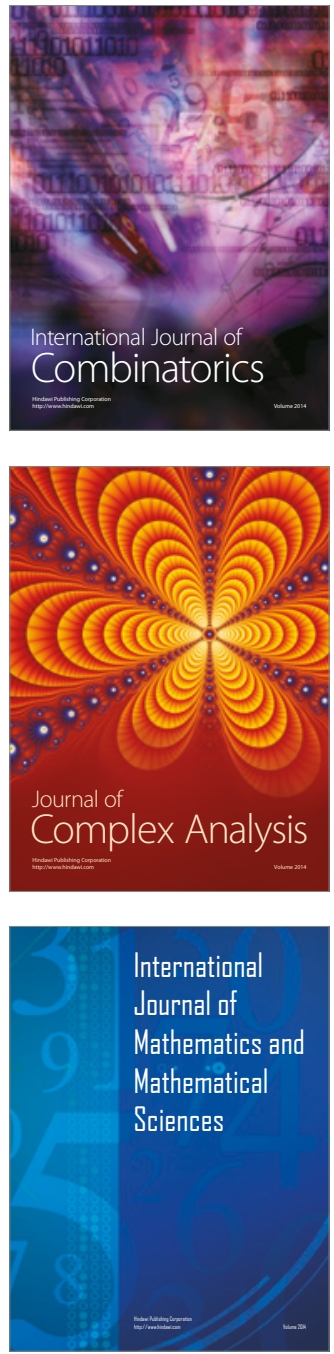
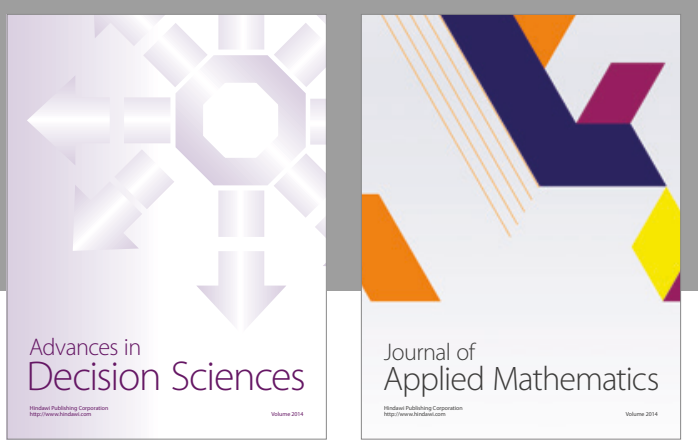

Algebra

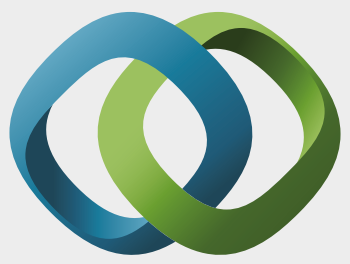

\section{Hindawi}

Submit your manuscripts at

https://www.hindawi.com
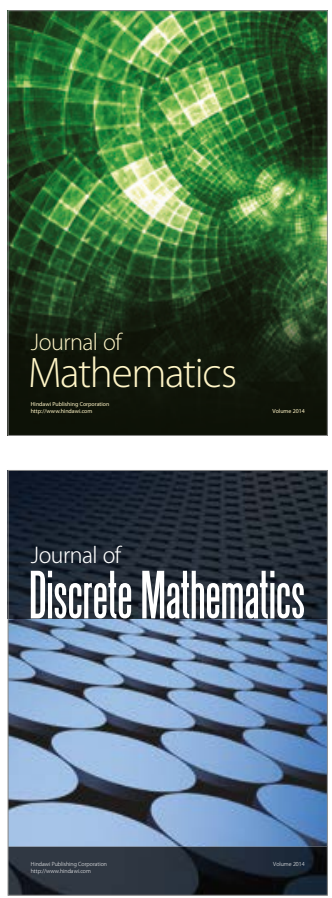

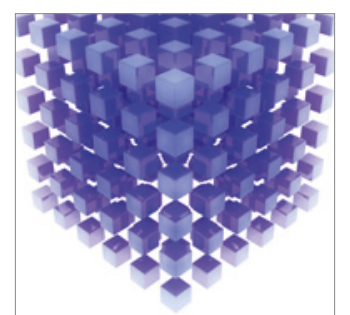

Mathematical Problems in Engineering
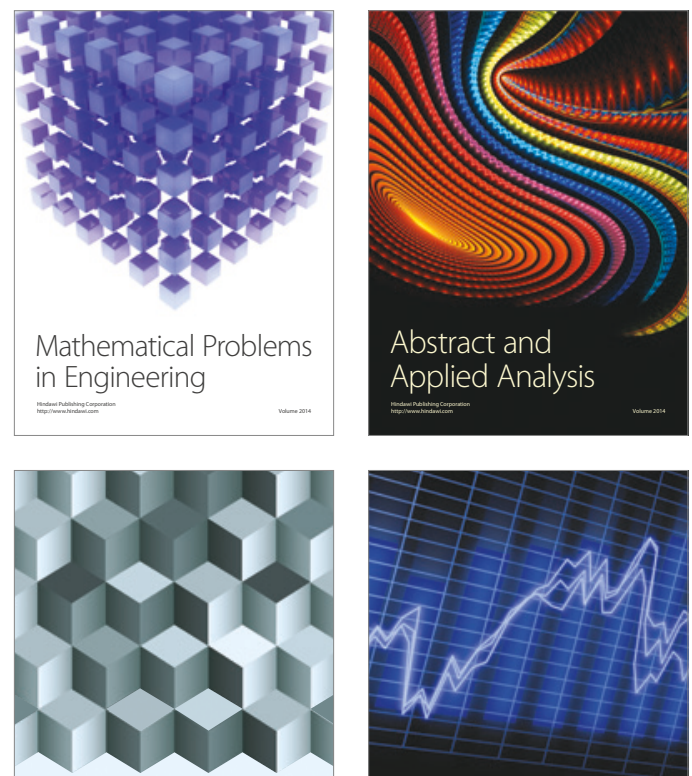

Journal of

Function Spaces

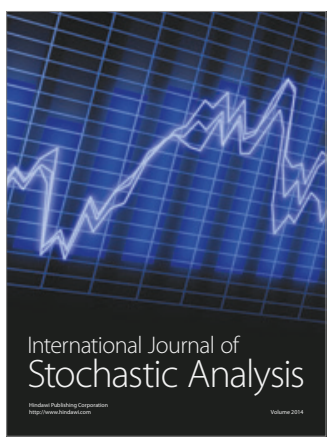

Probability and Statistics
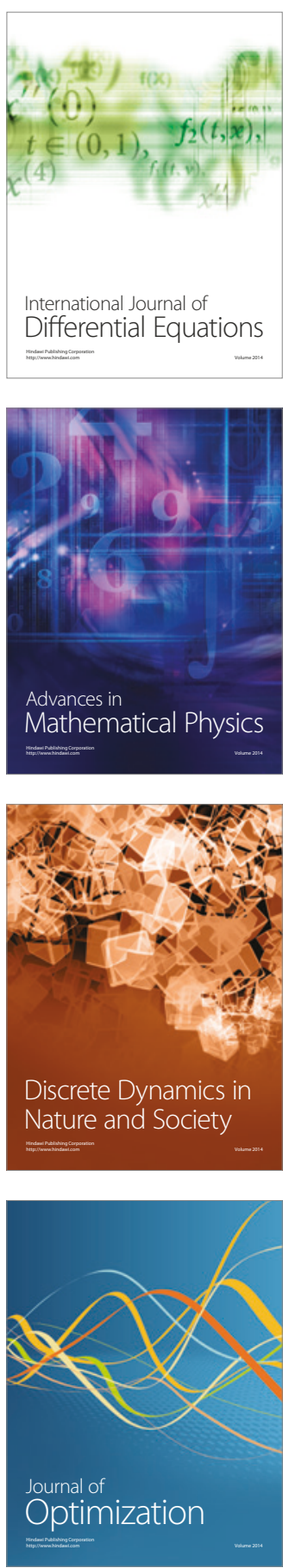